\section{Practice effects in the absolute judgment of frequency*}

\author{
MORTON A. HELLER and CARL AUERBACH \\ Yeshiva University, New York, N.Y. 10003
}

Two methods of training Ss to make absolute judgments of pitch are compared. Regular feedback, which requires the $S$ to name the note with which he is presented, and $A$ feedback, which requires the $S$ to judge whether the note with which he is presented is an $A$, both produce similar increases in performance. Apparently, labeling of the stimuli to be judged is not necessary for improvement of absolute judgments. Detailed analysis of the data suggest two learning mechanisms: Ss' judgments improve as a result of exposure to the range of the notes to be judged, and Ss appear to develop a sense of the range of the notes, and of the endpoints and midpoint of the range, so that they can place notes more accurately within the range. A-feedback training may, in addition, promote learning of notes in the immediate region of $A$.

Absolute judgment of frequency requires that Ss supply the correct musical name of a pure tone or of the fundamental frequency of a complex sound without immediate reference to an external standard of comparison. There is a good deal of evidence that Ss' ability to make absolute judgments of frequency can be improved by training with feedback, i.e., giving them information about the correct musical name of a note immediately after they make an absolute judgment (Neu, 1947).

Recently, Cuddy (1968) reported results from a different sort of training procedure. She compared two methods of training. A regular feedback condition (RFB) involved presenting Ss with the notes of which they have to make absolute judgments and telling them the name of the note after they make their response. An A-feedback (AFB) condition required Ss to judge whether the note with which they were presented was an $A$ and telling them whether they were correct. Cuddy found that the improvement of absolute judgment performance was greater for the AFB group than for the RFB group, which did not, in fact, improve at all.

Before one can attribute this puzzling result to the differences in training procedure, it must be the case that both groups were exposed to the same stimuli. In Cuddy's ex Deriment. they were not. The AFB group was trained with a "shaping" procedure, and the RFB group was not, so that the AFB group heard more As than did the RFB group. In addition, the AFB group was trained to a criterion of two or fewer errors, whereas the RFB group was merely presented with

* This research was supported by PHS Research Grant 1-RO 1-MH-16856-02 from the Behavioral Science Research Branch, National Institute of Mental Health. the stimulus sequence. Consequently, the AFB group got more training than did the RFB group.

The present replication of Cuddy's stimuli to which the two groups are exposed to see if the superiority of the AFB group would still be found.

\section{METHOD}

The experimental method is essentially that of Cuddy's study. The stimuli were 10 pure tones on the equal-tempered scale ranging from $F_{4}$ $(349 \mathrm{~Hz})$ to $D_{5}(587 \mathrm{~Hz})$, centered about $\mathrm{A}_{4}(440 \mathrm{~Hz})$ (Helmholtz, 1954). The tones were produced with a Krohn-Hite audio oscillator and monitored with a Beckman-Berkely frequency counter. They were recorded on tape to make up the training and testing sequences described below. In each sequence, the duration of a tone was 1 sec and the interval between tones was $10 \mathrm{sec}$. The recording of each tone began at a random point of the sinusoidal voltage which produced it. The frequency of the tones on the tape did not vary from the nominal value by more than $2 \mathrm{~Hz}$. The sequences thus prepared were played to the $S$ through Permoflux PDR 8 earphones at a sound-pressure level of roughly $50 \mathrm{~dB}$ on a Norelco Carrycorder 150 tape recorder.

Two groups of Ss were used: an A-feedback group (AFB), and a regular feedback (RFB) group. Each group consisted of eight Ss who had been recruited from a student population and randomly assigned to one of the two groups. The Ss had no special musical training.

The sequence of events during the (1) Familiarization-Ss were played a familiarization tape consisting of the 10-note scale $F_{4}$ to $D_{5}$ in ascending order. Each note was followed by its letter name. (2) Pretest-all 10 notes were presented eight times in a study was aimed at equating the experiment was as follows: random order, subject to the restriction that no note be repeated more than twice in succession. Ss were required to give the letter name of each note. There was no feedback after the notes. (3) Training-Ss were played four training tapes, each consisting of 80 randomly ordered presentations of the 10 notes of the scale, again subject to the restriction that no note be repeated more than twice in succession. The proportion of As on the first tape was 0.40 , on the second tape 0.30 , on the third tape 0.20 , and on the fourth tape 0.10 . The other notes occurred approximately equally often. The 10 -note familiarization tape was played prior to each training tape.

The Ss in the RFB group were asked to respond to each note immediately after it was presented by writing down its letter name. Five seconds after the termination of each note, a prerecorded voice gave the correct letter name of the note. AFB Ss were asked to respond to each note immediately after it was presented by writing down their judgment as to whether or not they judged the note to be an $A$. Five seconds after the termination of each note, a prerecorded voice said " $A$ " if the note was an $A$, and otherwise said nothing. (4) Post test-the 10 -note familiarization tape of the first part of the experiment was played, and then Ss were tested on tapes similar to those making up the pretest sequence. The pretest and posttest tapes differed only in the order of the stimuli. The test consisted of requiring Ss to write down the correct letter name of each note. As during the pretest, there was no feedback after the notes.

The entire experiment took 2 days. The first day consisted of the pretest and the first two training tapes. The second day consisted of the last two training tapes and the posttest.

\section{RESULTS}

The experiment had the form of a three-factor experiment, namely, training method (AFB vs RFB), time of testing (pretest vs posttest), and tones, with repeated measures on the last two factors. The performance measures were the percentage of correct responses to each tone and the mean judgment error in semitones on each tone. Figure 1 is a plot of percentage of correct responses on each tone before and after training for AFB and RFB Ss. An analysis of variance appropriate to the repeated-measures design (Winer, 1962) was done for both performance measures with equivalent results, except for the presence of a significant triple interaction. That for percentage of correct responses is given in Table 1.

The initial performance level of 

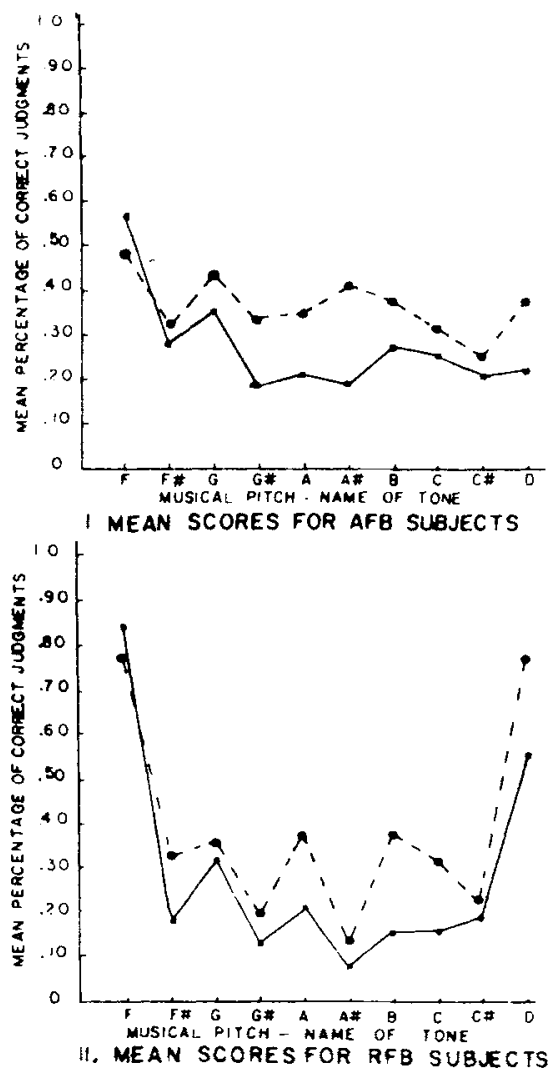

Fig. 1. Mean percentage of correct judgments per tone before and after training for AFB and RFB Ss. Solid lines represent pretest scores, and dotted lines represent posttest scores.

both groups was equivalent, and the groups improved equally with practice. This is shown statistically by the absence of both a main effect of training method and an interaction between training method and time of testing, together with the presence of a main effect of time of testing. The pretest and posttest percentage of correct responses were $27 \%$ vs $37 \%$ for the AFB group and $27 \%$ vs $38 \%$ for the RFB group. Considering mean judgment error in semitones, the pretest and posttest performances were 1.44 vs 1.00 for the AFB group and 1.40 vs 0.92 for the RFB group.

The significant main effect of tones implies that Ss' performance was better on some tones than on others. The Time of Testing by Tones interaction implies that Ss' performance improves more on some tones than on others. Both these facts are apparent from Fig. 1. One feature of the analysis of variance which suggests an effect of training method is the Training Method by Time of Testing interaction. This indicates that the two groups differ in their performance on specific tones. This difference is apparent in Fig. 1 , in which the curve for the RFB Ss is more bow-shaped than the curve for the AFB Ss. This difference is affected by the training procedure, as is shown by the significant triple interaction of Training Method by Time of Testing by Tones in the analysis of variance for mean judgment error. This is demonstrated by the relatively greater improvement of the AFB group on the tones immediately in the vicinity of $A$. DISCUSSION

The improvement in performance occurs equally for both the AFB and the RFB group. This suggests that Cuddy's results demonstrating the superiority of the AFB group were not due to differences between the methods of administering feedback, but rather to differences in the stimuli to which the groups were exposed.

The question remains as to why both groups improve. We suggest that Ss improve in part by developing a more accurate sense of the range of stimuli to be judged, so that they can place stimuli more accurately within this range. Several things suggest this view. The hypothesis is consistent with Ss' own reports of how they make their judgments. The hypothesis is also consistent with an early study of Wedell's (Wedell, 1934) which found that Ss trained with feedback to make

Table 1

Summary of Analysis of Variance

\begin{tabular}{lrrrl}
\hline \multicolumn{1}{c}{ Source } & SS & df & MS & F \\
\hline Between Ss & 4.06 & 15 & & \\
Training Method (A) & .07 & 1 & .07 & .24 \\
S Within Groups & 3.99 & 14 & .29 & \\
Within Ss & 20.77 & 304 & & \\
Time of Testing (B) & .84 & 1 & .84 & $\mathbf{7 . 0 0 * *}$ \\
A by B & .01 & 1 & .01 & 0.08 \\
B by St & 1.71 & 14 & .12 & \\
Tones (C) & 6.15 & 9 & .68 & $13.60^{* *}$ \\
A by C & $\mathbf{2 . 1 2}$ & 9 & .23 & $4.60^{* *}$ \\
C by S $\dagger$ & $\mathbf{5 . 7 2}$ & 126 & .05 & \\
B by C & .54 & 9 & .06 & $2.00^{* *}$ \\
A by B by C & .19 & 9 & .02 & 0.66 \\
B by C by S & 3.49 & 126 & .03 & \\
\hline
\end{tabular}

$*_{p}<.05, * * p<.01$, twithin groups
Table 2

Mean Judgment Error for Notes When They Follow Reference Notes F, A, or D Compared With Judgment Error on Same Notes When They Do Not Follow the Reference Notes*

\begin{tabular}{|c|c|c|c|}
\hline \multirow[b]{2}{*}{$\begin{array}{l}\text { Refe- } \\
\text { rence } \\
\text { Note }\end{array}$} & \multicolumn{2}{|c|}{$\begin{array}{l}\text { Mean Judgment Error } \\
\text { (in Semitones) }\end{array}$} & \multirow[b]{2}{*}{$\begin{array}{c}\text { Improve- } \\
\text { ment = } \\
(2)-(1)\end{array}$} \\
\hline & $\begin{array}{c}\text { (1) } \\
\text { Notes } \\
\text { Follow } \\
\text { Reference } \\
\text { Note }\end{array}$ & $\begin{array}{c}\text { (2) } \\
\text { Overall } \\
\text { Error } \\
\text { During } \\
\text { Posttest }\end{array}$ & \\
\hline $\mathbf{F}_{4}$ & 0.94 & 1.14 & $\begin{array}{l}.20 \\
\mathrm{p}\end{array}$ \\
\hline $\mathbf{A}_{4}$ & 0.96 & 1.08 & $\mathrm{p} \stackrel{.12}{<.10}$ \\
\hline $\mathrm{D}_{s}$ & 0.91 & 1.01 & $\mathrm{p}<.10$ \\
\hline
\end{tabular}

*A nalysis is for posttest only

absolute judgments of some notes in a given range of musical notes also increased their ability to make absolute judgments of the other notes in the range. Presumably, Ss improved by learning to place the notes presented to them in the range of notes and could thus better place notes with which they were not presented.

There is some internal evidence in our data consistent with the hypothesis that certain notes are important in developing or maintaining a sense of the range of notes to be judged. We suggest that exposure to notes at the midpoint and endpoints of the range, in this case $F_{4}$, $A_{4}$, and $D_{5}$, is particularly important in developing a sense of the range. An analysis was performed of the posttest data to see the effects of the hypothesized range defining notes on judgments subsequent to their presentation. As Table 2 shows, the mean judgment error on a note is less when it follows a range-defining note than when it does not. Although this effect is only significant for $F_{4}$, using a t test for paired correlated observations, it is nonetheless in the predicted direction in each case. This suggests that exposure to the hypothesized range-defining notes facilitates a temporary improvement in the sense of the range and thus increases the S's subsequent performance.

Cuddy's conclusion that RFB Ss do not improve is probably mistaken. The results reported here suggest this view. Moreover, inspection of Cuddy's original data reveals that four out of the five RFB Ss in Cuddy's study did improve, whereas the fifth showed a decrease in performance. Thus, most of the RFB Ss demonstrated an improvement in performance, although the "average S" showed no improvement. 
REFERENCES

CUDDY, L. L. Practice effects in the absolute judgment of pitch. Joumal of the Acoustical Society of America, 1968, 43, 1069-1076.

HELMHOLTZ, H. L. F. On the sensation of tone. New York: Dover, 1954.

NEU, D. M. A critical review of the literature on absolute pitch. Psychological Bulletin, 1947, 44, 249-268.

WEDELL, C. H. The nature of absolute judgment of pitch. Journal of
Experimental Psychology, 1934, 17, 458-508.

WINER, B. J. Statistical principles in experimental design. New York: McGraw-Hill, 1962. 